\title{
Theory of parabolic pulse generation in tapered fiber
}

\author{
Anton I. Latkin \\ Institute of Automation and Electrometry, Novosibirsk 630090, Russia \\ Sergei K. Turitsyn \\ Photonics Research Group, School of Engineering and Applied Science, Aston University, Birmingham B4 7ET, UK \\ Alexej A. Sysoliatin \\ Fiber Optics Research Center, General Physics Institute, Moscow 119991, Russia
}

Received September 11, 2006; accepted November 9, 2006; posted November 21, 2006 (Doc. ID 74933); published January 26, 2007

We examine similarities and differences between high-power parabolic pulse generation in an active medium and in tapered fiber with decreasing normal dispersion. Using a realistic tapered fiber design, we demonstrate the possibility of parabolic pulse generation without an external pump and determine the limitations of this approach. (c) 2007 Optical Society of America

OCIS codes: $060.2280,060.4370,060.5530,320.5540$.

High-power pulse propagation in optical fiber is strongly affected by nonlinear phenomena. Mastering these nonlinear effects can result in the development of new attractive techniques for optical signal generation, processing, and manipulation. In the normal dispersion regime, nonlinear-dominated optical pulse broadening generally leads to wave breaking that manifests itself as waveform steepening with subsequent growing oscillations at the pulse tails. However, there is an interesting class of breakingfree pulses with a parabolic profile in the energycontaining core that can propagate in a stable selfsimilar manner, holding a certain relation (scaling) among changing pulse power, width, and chirp. ${ }^{1}$ Such a parabolic pulse can be analytically described as an approximate solution of the nonlinear Schrödinger equation (NLSE) with normal dispersion and gain in the large-amplitude (or small-dispersion) limit. ${ }^{1-5}$ Note that the self-similar parabolic approximation is applied only in the central part of the pulse. A more accurate mathematical description that matches the parabolic core with the pulse tails has been presented in Ref. 6. Parabolic pulses (PPs) have recently attracted a great deal of attention because of their potential importance for high-power femtosecond lasers, ${ }^{7-9}$ applications in spectral broadening and supercontinuum generation, ${ }^{10,11}$ and all-optical signal processing and regeneration. ${ }^{12,13}$

Generation of PPs by using an optical fiber amplifying medium (e.g., Er-doped fiber amplifiers, Ybdoped fiber, or passive optical fibers with distributed Raman amplification) has been successfully demonstrated in a number of recent publications (see, e.g., Refs. 14 and 15 and references therein). Breakingfree optical pulse amplification is beneficial for highpower lasers and similar applications. However, there is a range of physical and technical problems (e.g., telecommunication signal processing) where high signal power is not required and the most valuable features are the specific parabolic pulse shape and chirp. An active medium, inevitably, introduces amplified spontaneous emission noise. Therefore, taking into account a variety of possible applications, it is of interest to examine other approaches to PP generation. A simple alternative method for generation of PPs in a passive device by means of a dispersion-decreasing fiber (DDF) was proposed in Ref. 16. The first experiments ${ }^{17,18}$ confirmed the possibility of PP generation in DDF. It was pointed out in Ref. 16 that in a system described by the ideal lossless NLSE with dispersion decreasing as $D(z)$ $=D(0) /\left(1+\Gamma_{0} z\right)$ (hyperbolic profile) optical pulse evolution is formally similar to that in active medium with a constant gain $\Gamma_{0}$. In this Letter we demonstrate that fiber loss and high-order dispersion breaks this similarity between tapered fiber and an active medium, substantially affecting PP generation. We present a theoretical analysis of the limitations imposed by higher-order dispersion and loss on the process of $\mathrm{PP}$ generation in a tapered fiber.

Decreasing dispersion can be achieved in a tapered fiber with an appropriately varying core diameter. ${ }^{19}$ Without loss of generality we consider throughout the Letter a normal dispersion tapered fiber fabricated from the dispersion-shifted fiber (DSF) (with the zero-dispersion point close to $1550 \mathrm{~nm}$ used in a recent experiment. ${ }^{18}$ Note that the zero-dispersion point depends on the fiber diameter and changes during the pulling process. Smaller absolute values of dispersion correspond to the larger core diameters (in the normal dispersion region). Therefore, for PP generation, light should be launched into the taper from the narrow edge as schematically shown in Fig. 1(a). Input and output diameters of the fiber can be tailored to achieve the desirable dispersion curve (i.e., hyperbolic profile). Typically, the difference of diameters is smaller than $10 \mu \mathrm{m}$ (with the DSF diameter of about $120 \mu \mathrm{m}$ ), and higher-order dispersion terms and fiber nonlinearity with a good accuracy can be considered to be constants along the fiber for a fixed 


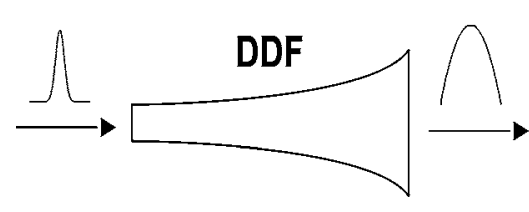

(a)

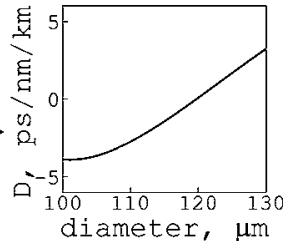

(b)

Fig. 1. (a) Experimental setup and (b) dispersion curve for tapered fiber.

wavelength. The typical dispersion-diameter curve for the pulled DSF at the wavelength of $1550 \mathrm{~nm}$ is shown in Fig. 1(b).

A dispersion curve is calculated by taking into account the practical refractive index profile for varying outer fiber diameters. Chromatic dispersion is computed by solving the scalar wave equation while using Sellmeyer coefficients for a fiber with a $\mathrm{SiO}_{2} / \mathrm{GeO}_{2}$ core. By selecting appropriate parameters of the refractive index profile (geometrical parameters, core/cladding refractive index, and so on) it is possible to fabricate fiber structure with optimized chromatic dispersion characteristics and spatial profiles. ${ }^{19}$ We consider in the simulations a hyperbolic dispersion profile along the taper fiber, starting with the value $D(0)=-2 \pi c \beta_{2} / \lambda_{0}^{2}=-2 \mathrm{ps} / \mathrm{nm} / \mathrm{km}$, giving the corresponding effective gain $\Gamma_{0} \simeq 4 \mathrm{~km}^{-1}$, nonlinear coefficient $\gamma=2 \mathrm{~W}^{-1} \mathrm{~km}^{-1}$, dispersion slope $S$ $=0.057 \mathrm{ps} / \mathrm{km} / \mathrm{nm}^{2}$, and fiber loss $\alpha=0.3 \mathrm{~dB} / \mathrm{km}$.

The master propagation equation for electrical field evolution along an optical fiber reads as

$$
i \frac{\partial A}{\partial z}-\frac{\beta_{2} d(z)}{2} \frac{\partial^{2} A}{\partial T^{2}}-i \frac{\beta_{3}}{6} \frac{\partial^{3} A}{\partial T^{3}}=-\gamma|A|^{2} A-i \frac{\alpha}{2} A .
$$

Here $A(z, t)$ is a slowly varying envelope of the pulse, $d(z)$ represents variation in the group-velocity dispersion $\beta_{2} d(z)$ due to tapering and is normalized such that $d(0)=1, \gamma$ is a nonlinear coefficient, $\beta_{3}$ is thirdorder dispersion (TOD), and $\alpha$ is fiber loss.

First, let us briefly recall, following Ref. 16, an analogy between an active medium and a DDF. Neglecting higher-order dispersion and loss and applying the following change of variables $\zeta(z)=\int_{0}^{z} d(s) \mathrm{d} s$, $q(\zeta, t)=A(z, t) / \sqrt{d(\zeta)}$, one can transform Eq. (1) into the NLSE with constant coefficients and effective gain depending on $\zeta$ :

$$
i \frac{\partial q}{\partial \zeta}-\frac{\beta_{2}}{2} \frac{\partial^{2} q}{\partial T^{2}}+\gamma|q|^{2} q=i \frac{\Gamma(\zeta)}{2} q .
$$

Here $\Gamma(\zeta)=-d^{\prime}(\zeta) / d(\zeta)$. The constant gain $\Gamma(\zeta)=\Gamma_{0}$ corresponds to the hyperbolic profile of dispersion $d(z)=1 /\left(1+\Gamma_{0} z\right)$. Assuming a hyperbolic dispersion profile, new variables can be found explicitly:

$$
\begin{aligned}
\zeta(z) & =\left(1 / \Gamma_{0}\right) \ln \left(1+\Gamma_{0} z\right), \\
q(\zeta, t) & =A(z, t) \sqrt{1+\Gamma_{0} z} \\
& =A(z, t) \exp \left(\Gamma_{0} \zeta / 2\right),
\end{aligned}
$$

with fiber length in the new variables to be $\zeta_{L}=\ln (1$ $\left.+\Gamma_{0} L\right) / \Gamma_{0}$. In Ref. 16 it was proposed that a highly nonlinear fiber with a hyperbolic profile of decreasing dispersion be used for generation of PPs. Here we discuss practical limitations of using tapered fiber for generation of parabolic pulses. In what follows we compare $\mathrm{PP}$ generation in DDF of length $L$ and in a corresponding active fiber with gain $\Gamma_{0}$ and length $\zeta_{L}$. Note that in the case of lossless DDF, mathematically, indeed, these two problems are the same: the initial pulse evolves into a PP when $L \rightarrow \infty$. However, as we show below, inclusion of the effects of higherorder dispersion and fiber loss breaks this analogy and imposes certain constraints on using tapered fiber for PP generation.

Next, we investigate the effects of TOD and fiber loss on PP generation in DDF. Making a change of variables $\zeta$ and $q$ as introduced above in the generalized NLSE (1) and assuming the hyperbolic dispersion profile yields the following effective propagation equation:

$$
\begin{aligned}
i \frac{\partial q}{\partial \zeta} & -\frac{\beta_{2}}{2} \frac{\partial^{2} q}{\partial T^{2}}-i \frac{\beta_{3}}{6} \exp \left(\Gamma_{0} \zeta\right) \frac{\partial^{3} q}{\partial T^{3}} \\
& =-\gamma|q|^{2} q+\frac{i}{2}\left[\Gamma_{0}-\alpha \exp \left(\Gamma_{0} \zeta\right)\right] q .
\end{aligned}
$$

Two important differences between NLSE-based models for conventional active media and Eq. (3) for DDF can be noted. First, in the case of DDF the TOD effect grows exponentially with distance and, even if the value of $\beta_{3}$ is small, the effect of higher-order dispersion will be critical at some propagation distance. Second, instead of constant gain in the DDF, in tapered fiber the effective gain decreases with propagation as shown in Fig. 2(a).

It is seen from the derived Eq. (3) that in DDF there is a critical distance $\zeta_{0}$ that separates regimes of the effective amplification and attenuation: $\zeta_{0}$ $=\ln \left(\Gamma_{0} / \alpha\right) / \Gamma_{0}$, or in the real world variables $L_{0}=1 / \alpha$ $-1 / \Gamma_{0} \simeq 1 / \alpha$, when $\Gamma_{0} \gg \alpha$. A simple estimate for typical values of DDF parameters indicates that for the purpose of $\mathrm{PP}$ generation the length of the DDF ultimately should not exceed $14 \mathrm{~km}\left(\zeta_{L} \approx 1 \mathrm{~km}\right)$. Figure 2(b) shows that, indeed, even in the ideal tapered fiber without TOD after propagation over approximately $\zeta_{L} \approx 1 \mathrm{~km}$ a nonlinear self-similar growth of a

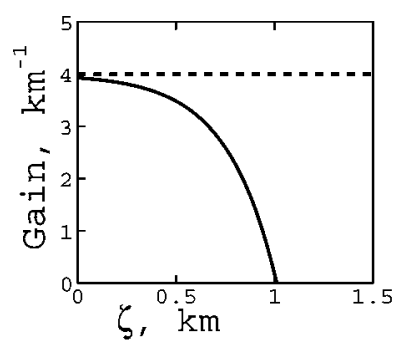

(a)

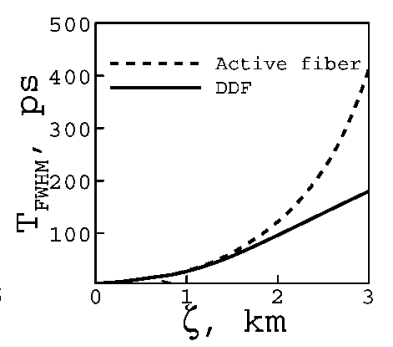

(b)
Fig. 2. Comparison of (a) the effective gain and (b) the pulse width in the DDF (solid curves) and corresponding active fiber (dashed curves). Here for both fibers the TOD is switched off to examine pure gain effects. 
pulse width corresponding to $\mathrm{PP}$ formation is replaced by linear dispersive broadening.

Next we verify the analytical results by direct numerical modeling and compare PP generation in DDF and in a corresponding active medium as introduced above. Figure 3 shows that in real DDF PP generation is possible only over a limited propagation distance. Here the left-hand column shows pulse and spectrum evolution in DDF [solution of Eq. (3)] and the right-hand column those in the corresponding active fiber. For convenience of comparison the results for DDF are presented with the new variables; therefore, the pulse amplitude is growing here. It is seen that after certain distance wave breaking due to TOD occurs, unlike the smoother PP generation in the equivalent active media. Note also that for a practical choice of taper fiber parameters disintegration of the PP starts at distances smaller than the limit $\zeta_{L}$ $\approx 1 \mathrm{~km}$ ) imposed by the effect of the depleting gain described above. Therefore the major limitations in this case are imposed by TOD, indeed. A possible way to relax the limitations imposed by the TOD is to use dispersion-flattened DDF.

In conclusion, we have examined similarities and differences between high-power parabolic pulse generation in an active medium and in tapered fiber with

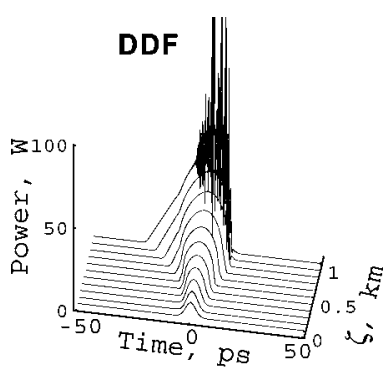

(a)

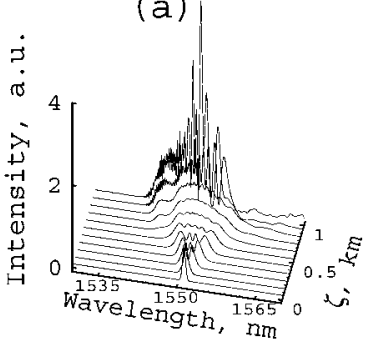

(c)

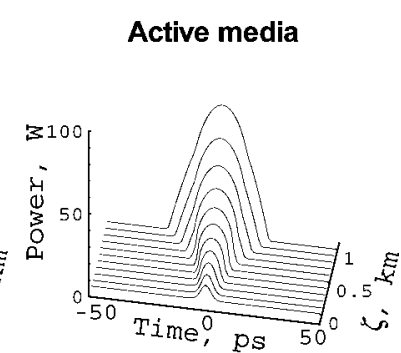

(b)

(d)

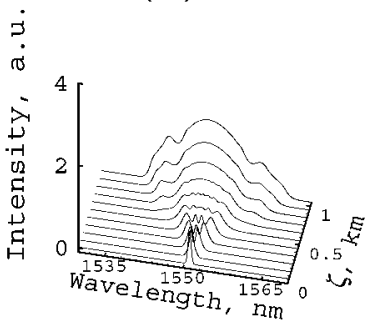

Fig. 3. (a) Pulse evolution in DDF (with TOD) and (b) in the equivalent active fiber. Corresponding spectrum evolution (c) in DDF and (d) in active fiber. decreasing normal dispersion. We have demonstrated that although PP generation in DDF is similar to the case of active fiber, the effect of TOD is critical for the DDF case, and it is mandatory either to apply a scheme with a very accurately designed taper fiber length or to use dispersion-flattened DDF.

A. Latkin's e-mail address is a.i.latkin@ngs.ru.

\section{References}

1. D. Anderson, M. Desaix, M. Karlson, M. Lisak, and M. L. Quiroga-Teixeiro, J. Opt. Soc. Am. B 10, 1185 (1993).

2. M. E. Fermann, V. I. Kruglov, B. C. Thomsen, J. M. Dudley, and J. D. Harvey, Phys. Rev. Lett. 84, 6010 (2000).

3. V. I. Kruglov, A. C. Peacock, J. M. Dudley, and J. D. Harvey, Opt. Lett. 25, 1753 (2000).

4. V. I. Kruglov, A. C. Peacock, J. M. Dudley, and J. D. Harvey, J. Opt. Soc. Am. B 19, 461 (2002).

5. V. I. Kruglov and J. D. Harvey, J. Opt. Soc. Am. B 23, 2541 (2006).

6. S. Boscolo, S. K. Turitsyn, V. Y. Novokshenov, and J. H. B. Nijhof, Theor. Math. Phys. 133, 1647 (2002).

7. J. Limpert, T. Schreiber, T. Clausnitzer, K. Zöllner, H. Fuchs, and E. Kley, Opt. Express 10, 628 (2002).

8. F. Ilday, F. Wise, and F. Kärtner, Opt. Express 12, 2731 (2004).

9. B. Ortaç, A. Hideur, M. Brunel, C. Chédot, J. Limpert, A. Tünnermann, and F. Ö. Ilday, Opt. Express 14, 6075 (2006).

10. K. Tamura, H. Kubota, and M. Nakazawa, IEEE J. Quantum Electron. 36, 773 (2000).

11. F. Parmigiani, C. Finot, K. Mukasa, M. Ibsen, M. A. Roelens, P. Petropoulos, and D. J. Richardson, Opt. Express 14, 7617 (2006).

12. K. Kikuchi, K. Taira, T. Sakamoto, and T. Tanemura, in 29th European Conference on Optical Communication (IEEE, 2003), paper We3.7.2.

13. S. Boscolo and S. K. Turitsyn, IEEE Photon. Technol. Lett. 17, 1235 (2005).

14. C. Finot, G. Millot, C. Billet, and J. M. Dudley, Opt. Express 11, 1547 (2003).

15. C. Finot, F. Parmigiani, P. Petropoulos, and D. Richardson, Opt. Express 14, 3161 (2006).

16. T. Hirooka and M. Nakazawa, Opt. Lett. 29, 1114 (2004).

17. B. Kibler, C. Billet, P. A. Lacourt, R. Ferriere, L. Larger, and J. M. Dudley, Electron. Lett. 42, 965 (2006)

18. A. Latkin, A. Sysoliatin, A. Plocky, P. Harper, J. Harrison, and S. K. Turitsyn, in 32nd European Conference on Optical Communication (IEEE, 2006), paper We2.3.2.

19. V. A. Bogatyrjov, M. M. Bubnov, E. M. Dianov, A. A. Sysoliatin, Pure Appl. Opt. 4, 345 (1995). 